SATURDAY, 17 JUNE 2017

HPR patients' perspectives, functioning and health (descriptive: qualitative or quantitative)

\section{SAT0719-HPR "AND SUDDENLY YOU ARE A PERSON AT RISK OF DEVELOPING RHEUMATOID ARTHRITIS!” DIFFERENT PERSPECTIVES OF INDIVIDUALS ON PREDICTIVE TESTING - RESULTS OF AN INTERNATIONAL QUALITATIVE INTERVIEW STUDY}

E. Mosor ${ }^{1,2}$, M. Stoffer ${ }^{2}$, G. Steiner ${ }^{2}$, K. Raza ${ }^{3}$, R.J. Stack ${ }^{3}$, G. Simons ${ }^{3}$, M. Falahee ${ }^{3}$, G. Schett ${ }^{4}$, A. Hueber ${ }^{4}$, M. Engelbrecht ${ }^{4}$, J. Smolen ${ }^{2}$, T. Stamm ${ }^{1}$ ${ }^{1}$ Section for Outcomes Research, CeMSIIS - Center for Medical Statistics, Informatics, and Intelligent Systems, Medical University of Vienna; ${ }^{2}$ Division of Rheumatology, Department of Internal Medicine III, Medical University of Vienna, Vienna, Austria; ${ }^{3}$ Department of Rheumatology, University of Birmingham and Sandwell \& West Birmingham Hospitals NHS Trust, Birmingham, United Kingdom; ${ }^{4}$ Rheumatology and Immunology, Department of Internal Medicine 3 , Universitätsklinikum Erlangen, Erlangen, Germany

Background: People at risk of developing rheumatoid arthritis (RA) may be candidates for interventions aimed at preventing RA development [1]. The identification of such "at risk" populations includes testing for genetic and other (e.g. autoantibody) biomarkers. However, little is known about the people's perspectives on these tests, how they react and cope when identified as being a person "at risk" and what their unmet needs are.

Objectives: To expore the perceptions about RA and predictive testing and to understand the various reactions and coping strategies used when identified as being at risk of developing RA from the perspective of those directly concerned. Methods: As part of the EuroTEAM project, a qualitative interview study with people who were informed of being at risk of developing RA was conducted. An interview schedule was developed and pilot-tested. Interviews were audiorecorded, transcribed verbatim and analyzed using thematic analysis.

Results: A total of 34 individuals (with rheumatoid factor and/or ACPA positive) already informed of being at risk of developing RA participated in the study, from Austria $(n=15)$, Germany $(n=15)$, and the UK $(n=4)$. In Vienna, people who had been tested within an expanded health exam participated in this study. In Birmingham and Erlangen, people with arthralgia were recruited from the outpatient clinics. Analysis of the interview data revealed five overarching themes related to predictive testing in the context of RA (Figure).

There were differences between the perceptions of althralgia patients and asymptomatic individuals. People suffering from pain were much more frightened and worried when informed of being at risk of developing RA. As a consequence, they modified their lives in a larger extent and had greater knowledge about RA than those without any symptoms who were rather surprised, kept calm and hardly changed their lifestyle after being tested positive. Allmost all participants in this study would appreciate precise predictive tests in the context of RA. However, more than half of them would refuse synovial biopsy (even if this could help quantify risk more accurately) or preventive medication. Recommendations for an improved procedure of predictive testing in the field of RA were given, which could promote uptake of preventive strategies.

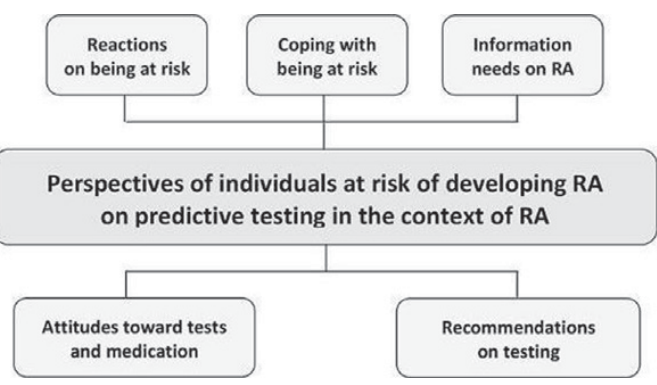

Conclusions: Participants showed large differences in views about predictive testing in the context of RA risk and offered specific suggestions that should be incorporated into service design and delivery in the context of future predictive testing programmes. These findings may also be relevant to prediction and prevention in the context of other diseases where multiple genetic risk factors interact with environmental risk factors to drive disease development.

References:

[1] van Steenbergen, H.W., T.W. Huizinga, and A.H. van der Helm-van Mil, The preclinical phase of rheumatoid arthritis: what is acknowledged and what needs to be assessed? Arthritis Rheum, 2013. 65(9): p. 2219-32.

Disclosure of Interest: None declared

DOI: 10.1136/annrheumdis-2017-eular.4705
SATURDAY, 17 JUNE 2017

HPR service developments, innovation and economics in healthcare

\section{SAT0720-HPR LONGITUDINAL ANALYSIS OF RESPONSE, COSTS AND RESOURCE USE OF PATIENTS WITH RHEUMATOID ARTHRITIS INITIATING BIOLOGIC DISEASE-MODIFYING ANTIRHEUMATIC DRUGS (BDMARDS) IN TAIWAN USING THE NATIONAL HEALTH INSURANCE RESEARCH DATABASE}

Q. Shi ${ }^{1}$, T. Treuer ${ }^{2}$, B.C. Wang ${ }^{3}$, C.L. Gaich ${ }^{4}$, C.-H. Lee ${ }^{5}$, W.-S. Wu ${ }^{5}$, W. Furnback ${ }^{3}$, K.-J. Li ${ }^{6}$, C.-H. Tang ${ }^{7}{ }^{1}$ Eli Lilly \& Co., Shanghai, China; ${ }^{2}$ Eli Lilly \& Co., Budapest, Hungary; ${ }^{3}$ Elysia Group Ltd., Taipei, Taiwan, Province of China; ${ }^{4}$ Eli Lilly \& Co., Indianapolis, United States; ${ }^{5}$ Eli Lilly \& Co; ${ }^{6}$ National Taiwan University; ${ }^{7}$ Taipei Medical University, Taipei, Taiwan, Province of China

Background: Rheumatoid arthritis (RA) is an inflammatory disorder associated with a significant physical and psychological burden. Patients with RA experience a lower quality of life than patients without RA. This study utilizes Taiwan's National Health Insurance Database (NHIRD) which is a claims-based database recording all interactions with the National Health Insurance $(\mathrm{NHI})$ system.

Objectives: The objective of this study was to use the NHIRD to estimate the percentage of newly treated patients with inadequate response (IR) to biologic disease-modifying antirheumatic drugs (bDMARDs) as well as the costs and resources.

Methods: Data were from the catastrophic illness file within the NHIRD from $1 / 1 / 2009$ to $12 / 31 / 2013$. The index period spanned 2010 with a pre-index period consisting of the index date -365 days, and patient follow-up was index date to 365 days post-index. Patients with a catastrophic illness card for RA were included in the study. Biologically-naïve patients included those who did not have a claim for a bDMARD or exclusion diagnoses in the pre-index period. Biologically-naïve patients with a bDMARD claim were indexed upon their first claim for a bDMARD during the index year. A validated algorithm was used to examine the rate of inadequate response in the biologically-naïve cohort of patients. Inadequate responders met one or more of the following criteria during their year of follow-up: low adherence (proportion of days covered $<0.80$ ); switched to or added a second bDMARD; added a new conventional synthetic DMARD (csDMARD); received $\geq 1$ glucocorticoid injection; or increased oral glucocorticoid dosing. All-cause mean annual direct costs and resource use were measured in the year of follow-up. Costs were converted from NT\$ to EUR using 1 NT $\$=0.03$ EUR.

Results: A total of 818 biologically-naïve patients initiated a bDMARD and were included in the study. At 1 year of follow-up, $66.01 \%(n=540)$ were classified as bDMARD-IR, 31.54\% ( $n=258)$ were classified as confirmed stable, and $2.44 \%$ $(\mathrm{n}=20)$ were of unknown response status. Mean annual total direct costs in the follow-up year were $€ 14,669$ and $€ 12,867$ for the confirmed stable and inadequate responder cohorts, respectively. Mean annual non-medication costs were $40.4 \%(€ 578)$ higher for the IR cohort, and mean annual medication costs were $17.2 \%(€ 2,379)$ higher for the confirmed stable cohort. IR patients had higher mean annual resource utilization in the follow-up year with increases in hospital admissions ( 0.46 vs. 0.1 ), hospital days (3.4 vs. 0.49), outpatient visits (42.50 vs. 37.66$)$, and emergency department visits ( 0.51 vs. 0.3 )

Conclusions: A significant proportion of patients with RA initiating a bDMARD for the first time showed an IR to their treatment within a year. Patients with an IR had increased resource utilization and higher non-medication costs than those with stable disease. This level of IR suggests an unmet need in the RA treatment paradigm.

Disclosure of Interest: Q. Shi Employee of: Eli Lilly \& Co., T. Treuer Employee of: Eli Lilly \& Co., B. Wang Consultant for: Eli Lilly \& Co., C. Gaich Employee of: Eli Lilly \& Co., C.-H. Lee Employee of: Eli Lilly \& Co., W.-S. Wu Employee of: Eli Lilly \& Co., W. Furnback Consultant for: Eli Lilly \& Co., K.-J. Li: None declared, C. $-\mathrm{H}$. Tang: None declared

DOI: 10.1136/annrheumdis-2017-eular.5315

SATURDAY, 17 JUNE 2017

HPR epidemiology and public health (including prevention)

\section{SAT0721-HPR EXPOSURE TO PASSIVE SMOKING AND RA RISK; RESULTS FROM THE SWEDISH EIRA STUDY}

A.K. Hedström ${ }^{1}$, L. Klareskog ${ }^{2}$, L. Alfredsson ${ }^{1} .{ }^{1}$ Institute of Environmental Medicine; ${ }^{2}$ Department of Medicine, Karolinska Institutet, Stockholm, Sweden

Background: Smoking has consistently been associated with increased risk of developing rheumatoid arthritis (RA). However, no studies have been performed investigating the effect of exposure to environmental tobacco smoke (i.e. passive smoking) on RA risk.

Objectives: The aim of this study was to estimate the influence of passive smoking on the risk of developing the two major subsets of RA, defined by anti-citrullinated protein antibodies (ACPA) status. 